\title{
Pretreatment C-reactive Protein to Albumin Ratio Predicts Clinical Outcomes in Patients with Peripheral T-cell Lymphoma
}

Jongheon Jung

National Cancer Center

Ja Yoon Heo

National Health Insurance Service Ilsan Hospital

Eunyoung Lee

National Cancer Center

Hyewon Lee

National Cancer Center

Myung Hee Chang

National Health Insurance Service Ilsan Hospital

Ju-Hyun Park

Dongguk University

Hyeon-Seok Eom ( $\nabla$ hseom@ncc.re.kr)

National Cancer Center

\section{Research Article}

Keywords: peripheral T-cell lymphoma, C-reactive protein, albumin, prognosis, survival

Posted Date: January 13th, 2022

DOI: https://doi.org/10.21203/rs.3.rs-1240904/v1

License: (c) (i) This work is licensed under a Creative Commons Attribution 4.0 International License. Read Full License 


\section{Abstract}

Peripheral T-cell lymphoma (PTCL) is an aggressive and heterogenous T-cell lymphoid malignancy. The prognostic value of C-reactive protein-to-albumin ratio (CAR) has never been assessed in PTCL. This study retrospectively reviewed the medical records of 76 patients diagnosed with various subtypes of PTCL. The value of 0.794 was identified as the most discriminative point of CAR, and clinical outcomes, including response rate, overall survival (OS), and progression-free survival (PFS), were compared between the high $(>0.794, n=25)$ and low $(\leq 0.794, n=51)$ CAR groups. After induction therapy, complete response was achieved in 39 patients (76.5\%) and 8 patients $(32.0 \%)$ in the low and high CAR groups, respectively $(p<0.001)$. During the median follow-up of 57.5 months, the high CAR group had significantly worse 5 -year PFS $(6.6 \%$ vs. $43.8 \%, p<0.0001)$ and 5 -year OS $(20.2 \%$ vs. $62.2 \%, p<0.0001)$ rates. With adjustment for the International Prognostic Index ( $\geq 3)$, Prognostic Index for PTCL-unspecified ( $\geq 3)$, and T cell score ( $\geq 2$ ), high CAR remained a significant prognostic factor for PFS (hazard ratio [HR]: 4.01, 95\% confidence interval [Cl] 2.04-7.86, p<0.001) and OS (HR: 2.97, 95\% Cl: 1.33-6.64, p=0.008). CAR might play a complementary role in predicting prognosis in patients with $\mathrm{PTCL}$, considering its simplicity, objectivity, and easy accessibility.

\section{Introduction}

Peripheral T-cell lymphomas (PTCLs) are a category of heterogeneous neoplasms that constitute 10$15 \%$ of non-Hodgkin's lymphomas[1]. Combination chemotherapy such as $\mathrm{CHO}$ (E)P (cyclophosphamide, doxorubicin, vincristine, and prednisone [plus etoposide]) is generally required as an initial treatment, and high-dose chemotherapy with autologous hematopoietic stem cell transplantation (HSCT) could be offered for a selected population of patients[2]. Most patients with PTCL, not otherwise specified (PTCLNOS) and angioimmunoblastic T-cell lymphoma (AITL) have been reported to present with advanced stage disease at diagnosis, and their survival rate was limited to between 30-45\%[3]. Importantly, the prognosis of anaplastic large cell lymphoma (ALCL) is largely dependent on the presence of anaplastic lymphoma kinase (ALK). In fact, one study found that the 5-year survival rate in ALK-negative ALCL patients was $49 \%$, which was significantly worse than that among ALK-positive ALCL patients (70\%)[4]. Although new strategies have been developed to improve the prognosis of PTCL, the majority of patients with PTCL do not achieve favorable clinical outcomes despite aggressive treatment strategies.

Traditionally, the International Prognostic Index (IPI), which is based on information for age (>60 years), stage (III or IV), increased serum lactate dehydrogenase (LDH) levels, Eastern Cooperative Oncology Group (ECOG) performance status $(>1)$, and the number of extranodal involvement $(>1)$, has been used as a prognostic marker for PTCL[5]. While many studies have demonstrated its usefulness in predicting clinical outcomes, there have been some objections for its use in certain types of PTCL[6]. Other prognostic tools, such as the Prognostic Index for PTCL-unspecified (PIT), which is based on information for age (>60 years), increased LDH, ECOG performance status (>1), bone marrow involvement, and the T cell score developed by the International T cell Project Network that defined advanced stage III or IV, as well as ECOG performance status $(>1)$, serum albumin $(<3.5 \mathrm{~g} / \mathrm{dl})$, and absolute neutrophil count $(6.5 \mathrm{x}$ 
$\left.10^{9} / \mathrm{I}\right)$, have been proposed, but their uses are generally limited for PTCL-NOS[7, 8]. Therefore, there is still a demand for an accurate and simple parameter for this heterogeneous group of aggressive diseases.

The C-reactive protein (CRP) to albumin ratio (CAR) has been investigated as a prognostic marker for various malignancies and has proven clinical value[9]. The availability of CAR has also been demonstrated in diffuse large B-cell lymphoma and extranodal natural killer T-cell lymphoma[10, 11]. Additionally, various blood-based biomarkers, such as platelets, serum globulin, ferritin, neutrophils, and lymphocytes, have been verified to facilitate prognostication[12, 13]. Importantly, these markers have been shown to reflect the tumor microenvironment and offer an easily accessible and objective index. However, the values of these markers have not yet been assessed in PTCL. Therefore, in this study, we evaluated the prognostic significance of these biomarkers in patients with newly diagnosed PTCL.

\section{Methods}

\section{Patient population}

In this study, we reviewed the medical records of 76 patients who were diagnosed with PTCL, including PTCL-NOS, AITL, and ALCL, that were either ALK positive or ALK negative. The diagnosis of all patients was histologically confirmed and they received combination chemotherapy as an induction treatment between 2007 and 2019 at the National Cancer Center in Korea and at the National Health Insurance Service Ilsan Hospital, Korea. Patients aged $>18$ years at diagnosis and who had available clinical and laboratory data at baseline before the initiation of treatment were included. The exclusion criteria were those with a lack of available medical records and those who did not receive chemotherapy. Medical records were retrospectively reviewed. The responses to chemotherapy were stratified based on the Lugano classification, which includes complete response (CR), partial response (PR), stable disease (SD), and progressive disease (PD)[14].

\section{Ethics Statement}

The study protocol was approved by the Institutional Review Board (IRB) (National Cancer Center 20200110, National Health Insurance Service Ilsan Hospital 2020-04-020) and complied with the Declaration of Helsinki. The requirement for informed consent was waived by the IRB, considering that no intervention was involved due to the retrospective nature of this study.

\section{Laboratory testing}

The initial values of CRP and albumin were collected using laboratory data from the day closest to the beginning of induction therapy. CAR was calculated as CRP divided by albumin. Other blood-based values, such as hemoglobin, platelet, neutrophil count, lymphocyte count, serum globulin, beta-2 microglobulin, ferritin, and lactate dehydrogenase (LDH), were also obtained from the patient's medical 
records. All laboratory data were obtained from the XE-2100 system (Sysmex, Kobe, Japan) at the National Cancer Center, Korea and at the National Health Insurance Service Ilsan Hospital, Korea.

\section{Statistical analysis}

Overall survival (OS) was defined as the time from the start of induction therapy to death from any cause or the last available follow-up date. Progression-free survival (PFS) was calculated from the first day of therapy to the date of disease progression or death. To investigate an appropriate CAR cutoff value for patients, the cutoff finder method described by Budczies et al. was applied[15]. Using this method, the optimal cutoff of CAR that split the results in the most significant log-rank test from the survival analysis was designated as 0.794 . The same method was used to define appropriate values for albumin-globulin ratio (AGR), beta-2 microglobulin, neutrophil-to-lymphocyte ratio (NLR), platelet-to-lymphocyte ratio (PLR), and ferritin.

Based on the cutoff value of CAR, patients were classified into high and low CAR groups, and their baseline characteristics were compared. Continuous variables were analyzed using the two-sample t-test or the Mann-Whitney $U$ test, depending on the results of the normality test. Categorical values were analyzed using the $\chi^{2}$ test or Fisher's exact test, as appropriate. Survival analyses were performed using the Kaplan-Meier method and log-rank test. Univariate and multivariate Cox proportional hazards models were applied to evaluate prognostic values, and hazard ratios (HRs) and 95\% confidence intervals (Cls) were obtained. Differences were considered significant at a two-sided $p$ value of $<0.05$. All statistical analyses were performed using R software (version 3.5.1; R Foundation for Statistical Computing, Vienna, Austria).

\section{Results}

\section{Patient characteristics}

In total, 76 patients with available data were included in this study. All patients were histologically diagnosed with PTCL and received chemotherapy as induction therapy. Of these, 25 (32.9\%) patients were classified into the high CAR group, while 51 (67.1\%) patients were classified into the low CAR group. Baseline characteristics of both groups are presented in Table 1. The most common diagnosis was PTCLNOS ( $n=31,40.8 \%)$, followed by AITL $(n=24,31.6 \%)$, ALK-negative ALCL $(n=12,15.8 \%)$, and ALK-positive ALCL $(n=9,11.8 \%)$. The high CAR group showed a significantly higher proportion of patients with advanced stage, poor performance status, elevated $L D H$, and extranodal involvement $(>1)$, which are all components of the IPI. The percentage of patients with B-symptoms at diagnosis was also significantly higher in the high CAR group than in the low CAR group ( $72.0 \%$ vs. $29.4 \%, p<0.001)$. More than two-thirds of the high CAR group initially had bone marrow involvement, while only approximately $20 \%$ of the low CAR group presented with it. The distributions of IPI, PIT, and T cell scores revealed significant differences between the two groups, as all factors predicted favorable outcomes for the low CAR group. Importantly, 
the regimens of induction therapy and the number of patients for whom autologous HSCT was performed were not significantly different between the groups. 
Table 1

Baseline characteristics of patients with peripheral T cell lymphoma.

\begin{tabular}{|c|c|c|c|c|c|}
\hline \multicolumn{2}{|l|}{ Variable } & \multirow{2}{*}{ 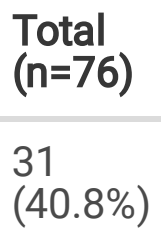 } & \multirow{2}{*}{$\begin{array}{l}\text { Low CAR }(n=51) \\
24(47.1 \%)\end{array}$} & \multirow{2}{*}{$\begin{array}{l}\text { High CAR }(n=25) \\
7(28.0 \%)\end{array}$} & \multirow{2}{*}{$\begin{array}{l}\begin{array}{l}\text { p- } \\
\text { value }\end{array} \\
0.236\end{array}$} \\
\hline Diagnosis & PTCL-NOS & & & & \\
\hline & AITL & $\begin{array}{l}24 \\
(31.6 \%)\end{array}$ & $14(27.5 \%)$ & $10(40.0 \%)$ & \\
\hline & $\begin{array}{l}\text { ALCL, } \\
\text { ALK- }\end{array}$ & $\begin{array}{l}12 \\
(15.8 \%)\end{array}$ & $6(11.8 \%)$ & $6(24.0 \%)$ & \\
\hline & $\begin{array}{l}\text { ALCL, } \\
\text { ALK+ }\end{array}$ & $\begin{array}{l}9 \\
(11.8 \%)\end{array}$ & $7(13.7 \%)$ & $2(8.0 \%)$ & \\
\hline \multirow[t]{2}{*}{ Sex } & Male & $\begin{array}{l}52 \\
(68.4 \%)\end{array}$ & $33(64.7 \%)$ & 19 (76.0\%) & 0.433 \\
\hline & Female & $\begin{array}{l}24 \\
(31.6 \%)\end{array}$ & $18(35.3 \%)$ & $6(24.0 \%)$ & \\
\hline \multirow[t]{2}{*}{ Age } & $>60$ & $\begin{array}{l}34 \\
(44.7 \%)\end{array}$ & $24(47.1 \%)$ & $10(40.0 \%)$ & 0.628 \\
\hline & $\leq 60$ & $\begin{array}{l}42 \\
(55.3 \%)\end{array}$ & 27 (52.9\%) & $15(60.0 \%)$ & \\
\hline \multirow[t]{2}{*}{ Stage } & $1-2$ & $\begin{array}{l}26 \\
(34.2 \%)\end{array}$ & $23(45.1 \%)$ & $3(12.0 \%)$ & 0.005 \\
\hline & $3-4$ & $\begin{array}{l}50 \\
(65.8 \%)\end{array}$ & 28 (54.9\%) & 22 (88.0\%) & \\
\hline \multirow[t]{2}{*}{ ECOG PS } & 0,1 & $\begin{array}{l}61 \\
(80.3 \%)\end{array}$ & 47 (92.2\%) & $14(56.0 \%)$ & $<0.001$ \\
\hline & $>1$ & $\begin{array}{l}15 \\
(19.7 \%)\end{array}$ & 4 (7.8\%) & $11(44.0 \%)$ & \\
\hline \multirow[t]{2}{*}{ B-Symptom } & Present & $\begin{array}{l}33 \\
(43.4 \%)\end{array}$ & 15 (29.4\%) & 18 (72.0\%) & $<0.001$ \\
\hline & None & $\begin{array}{l}43 \\
(56.6 \%)\end{array}$ & $36(70.6 \%)$ & 7 (28.0\%) & \\
\hline
\end{tabular}

Data are presented as number (frequency). CAR, C-reactive protein-to-albumin ratio; PTCL-NOS, peripheral T-cell lymphoma-not otherwise specified; AITL, angioimmunoblastic T-cell lymphoma; ALCL, anaplastic large cell lymphoma; ALK, anaplastic lymphoma kinase; ECOG PS, Eastern Cooperative Oncology Group performance status; LDH, lactate dehydrogenase; EN, extranodal involvement; BM, bone marrow; IPI, International Prognostic Index; PIT, prognostic index for PTCL-unspecified; CHO(E)P, cyclophosphamide, doxorubicin, vincristine, and prednisone (plus etoposide); ICED, ifosfamide, carboplatin, etoposide, dexamethasone; IMEP, ifosfamide, methotrexate, etoposide, prednisone; ESHAOx, etoposide, methylprednisolone, high-dose cytarabine, oxaliplatin; CR, complete response; PR, partial response; SD, stable disease; PD, progressive disease; HSCT, hematopoietic stem cell transplantation 


\begin{tabular}{|c|c|c|c|c|c|}
\hline \multicolumn{2}{|l|}{ Variable } & \multirow{2}{*}{$\begin{array}{l}\begin{array}{l}\text { Total } \\
(n=76)\end{array} \\
35 \\
(46.1 \%)\end{array}$} & \multirow{2}{*}{$\begin{array}{l}\text { Low CAR }(n=51) \\
31(60.8 \%)\end{array}$} & \multirow{2}{*}{$\begin{array}{l}\text { High CAR }(n=25) \\
4(16.0 \%)\end{array}$} & \multirow{2}{*}{$\begin{array}{l}\begin{array}{l}\text { p- } \\
\text { value }\end{array} \\
<0.001\end{array}$} \\
\hline LDH & Normal & & & & \\
\hline & Elevated & $\begin{array}{l}41 \\
(53.9 \%)\end{array}$ & $20(39.2 \%)$ & $21(84.0 \%)$ & \\
\hline \multirow[t]{2}{*}{ EN } & $\leq 1$ & $\begin{array}{l}49 \\
(64.5 \%)\end{array}$ & 37 (72.5\%) & $12(48.0 \%)$ & 0.044 \\
\hline & $>1$ & $\begin{array}{l}27 \\
(35.5 \%)\end{array}$ & $14(27.5 \%)$ & $13(52.0 \%)$ & \\
\hline \multirow[t]{2}{*}{$\begin{array}{l}\text { BM } \\
\text { involvement }\end{array}$} & $\begin{array}{l}\text { Not } \\
\text { involved }\end{array}$ & $\begin{array}{l}49 \\
(64.5 \%)\end{array}$ & $41(80.4 \%)$ & $8(32.0 \%)$ & $<0.001$ \\
\hline & Involved & $\begin{array}{l}27 \\
(35.5 \%)\end{array}$ & $10(19.6 \%)$ & $17(68.0 \%)$ & \\
\hline \multirow[t]{6}{*}{ IPI } & 0 & $\begin{array}{l}10 \\
(13.2 \%)\end{array}$ & $9(17.6 \%)$ & $1(4.0 \%)$ & $<0.001$ \\
\hline & 1 & $\begin{array}{l}14 \\
(18.4 \%)\end{array}$ & $14(27.5 \%)$ & $0(0.0 \%)$ & \\
\hline & 2 & $\begin{array}{l}17 \\
(22.4 \%)\end{array}$ & $13(25.5 \%)$ & $4(16.0 \%)$ & \\
\hline & 3 & $\begin{array}{l}20 \\
(26.3 \%)\end{array}$ & $10(19.6 \%)$ & $10(40.0 \%)$ & \\
\hline & 4 & $\begin{array}{l}8 \\
(10.5 \%)\end{array}$ & 2 (3.9\%) & $6(24.0 \%)$ & \\
\hline & 5 & $\begin{array}{l}7 \\
(9.2 \%)\end{array}$ & 3 (5.9\%) & $4(16.0 \%)$ & \\
\hline \multirow[t]{3}{*}{ PIT } & 0 & $\begin{array}{l}14 \\
(18.4 \%)\end{array}$ & 12 (23.5\%) & 2 (8.0\%) & $<0.001$ \\
\hline & 1 & $\begin{array}{l}27 \\
(35.5 \%)\end{array}$ & 25 (49.0\%) & 2 (8.0\%) & \\
\hline & 2 & $\begin{array}{l}14 \\
(18.4 \%)\end{array}$ & $7(13.7 \%)$ & $7(28.0 \%)$ & \\
\hline
\end{tabular}

Data are presented as number (frequency). CAR, C-reactive protein-to-albumin ratio; PTCL-NOS, peripheral T-cell lymphoma-not otherwise specified; AITL, angioimmunoblastic T-cell lymphoma; ALCL, anaplastic large cell lymphoma; ALK, anaplastic lymphoma kinase; ECOG PS, Eastern Cooperative Oncology Group performance status; LDH, lactate dehydrogenase; EN, extranodal involvement; BM, bone marrow; IPI, International Prognostic Index; PIT, prognostic index for PTCL-unspecified; CHO(E)P, cyclophosphamide, doxorubicin, vincristine, and prednisone (plus etoposide); ICED, ifosfamide, carboplatin, etoposide, dexamethasone; IMEP, ifosfamide, methotrexate, etoposide, prednisone; ESHAOx, etoposide, methylprednisolone, high-dose cytarabine, oxaliplatin; CR, complete response; PR, partial response; SD, stable disease; PD, progressive disease; HSCT, hematopoietic stem cell transplantation 


\begin{tabular}{|c|c|c|c|c|c|}
\hline \multicolumn{2}{|l|}{ Variable } & \multirow{2}{*}{$\begin{array}{l}\begin{array}{l}\text { Total } \\
(\mathrm{n}=76)\end{array} \\
14 \\
(18.4 \%)\end{array}$} & \multirow{2}{*}{$\begin{array}{l}\text { Low CAR }(n=51) \\
6(11.8 \%)\end{array}$} & \multirow{2}{*}{$\begin{array}{l}\text { High CAR }(n=25) \\
8(32.0 \%)\end{array}$} & \multirow{2}{*}{$\begin{array}{l}\mathrm{p}- \\
\text { value }\end{array}$} \\
\hline & 3 & & & & \\
\hline & 4 & $\begin{array}{l}7 \\
(9.2 \%)\end{array}$ & $1(2.0 \%)$ & $6(24.0 \%)$ & \\
\hline \multirow[t]{5}{*}{ T cell score } & 0 & $\begin{array}{l}19 \\
(25.0 \%)\end{array}$ & 19 (37.3\%) & $0(0.0 \%)$ & $<0.001$ \\
\hline & 1 & $\begin{array}{l}30 \\
(39.5 \%)\end{array}$ & $22(43.1 \%)$ & 8 (32.0\%) & \\
\hline & 2 & $\begin{array}{l}13 \\
(17.1 \%)\end{array}$ & $6(11.8 \%)$ & $7(28.0 \%)$ & \\
\hline & 3 & $\begin{array}{l}13 \\
(17.1 \%)\end{array}$ & 4 (7.8\%) & $9(36.0 \%)$ & \\
\hline & 4 & $\begin{array}{l}1 \\
(1.3 \%)\end{array}$ & 0 (0.0\%) & $1(4.0 \%)$ & \\
\hline \multirow[t]{4}{*}{ Induction } & $\mathrm{CHO}(\mathrm{E}) \mathrm{P}$ & $\begin{array}{l}46 \\
(60.5 \%)\end{array}$ & $33(64.7 \%)$ & $13(52.0 \%)$ & 0.513 \\
\hline & ICED & $\begin{array}{l}19 \\
(25.0 \%)\end{array}$ & 11 (21.6\%) & 8 (32.0\%) & \\
\hline & IMEP & $\begin{array}{l}9 \\
(11.8 \%)\end{array}$ & 5 (9.8\%) & $4(16.0 \%)$ & \\
\hline & ESHAOx & $\begin{array}{l}2 \\
(2.6 \%)\end{array}$ & 2 (3.9\%) & $0(0.0 \%)$ & \\
\hline \multirow[t]{4}{*}{ Response } & $\mathrm{CR}$ & $\begin{array}{l}47 \\
(61.8 \%)\end{array}$ & 39 (76.5\%) & 8 (32.0\%) & $<0.001$ \\
\hline & PR & $\begin{array}{l}7 \\
(9.2 \%)\end{array}$ & 3 (5.9\%) & $4(16.0 \%)$ & \\
\hline & SD & $\begin{array}{l}2 \\
(2.6 \%)\end{array}$ & 2 (3.9\%) & $0(0.0 \%)$ & \\
\hline & PD & $\begin{array}{l}17 \\
(22.4 \%)\end{array}$ & $6(11.8 \%)$ & $11(44.0 \%)$ & \\
\hline
\end{tabular}

Data are presented as number (frequency). CAR, C-reactive protein-to-albumin ratio; PTCL-NOS, peripheral T-cell lymphoma-not otherwise specified; AITL, angioimmunoblastic T-cell lymphoma; ALCL, anaplastic large cell lymphoma; ALK, anaplastic lymphoma kinase; ECOG PS, Eastern Cooperative Oncology Group performance status; LDH, lactate dehydrogenase; EN, extranodal involvement; BM, bone marrow; IPI, International Prognostic Index; PIT, prognostic index for PTCL-unspecified; CHO(E)P, cyclophosphamide, doxorubicin, vincristine, and prednisone (plus etoposide); ICED, ifosfamide, carboplatin, etoposide, dexamethasone; IMEP, ifosfamide, methotrexate, etoposide, prednisone; ESHAOx, etoposide, methylprednisolone, high-dose cytarabine, oxaliplatin; CR, complete response; PR, partial response; SD, stable disease; PD, progressive disease; HSCT, hematopoietic stem cell transplantation 


\begin{tabular}{|c|c|c|c|c|c|}
\hline \multicolumn{2}{|l|}{ Variable } & \multirow{2}{*}{$\begin{array}{l}\begin{array}{l}\text { Total } \\
(n=76)\end{array} \\
3 \\
(3.9 \%)\end{array}$} & \multirow{2}{*}{$\begin{array}{l}\text { Low CAR }(n=51) \\
1(2.0 \%)\end{array}$} & \multirow{2}{*}{$\begin{array}{l}\text { High CAR }(n=25) \\
2(8.0 \%)\end{array}$} & \multirow{2}{*}{$\begin{array}{l}\mathrm{p}- \\
\text { value }\end{array}$} \\
\hline & $\begin{array}{l}\text { Not } \\
\text { evaluated }\end{array}$ & & & & \\
\hline \multirow[t]{2}{*}{$\begin{array}{l}\text { Autologous } \\
\text { HSCT }\end{array}$} & $\begin{array}{l}\text { Not } \\
\text { performed }\end{array}$ & $\begin{array}{l}54 \\
(71.1 \%)\end{array}$ & 34 (66.7\%) & $20(80.0 \%)$ & 0.288 \\
\hline & Performed & $\begin{array}{l}22 \\
(28.9 \%)\end{array}$ & $17(33.3 \%)$ & 5 (20.0\%) & \\
\hline \multicolumn{6}{|c|}{$\begin{array}{l}\text { Data are presented as number (frequency). CAR, C-reactive protein-to-albumin ratio; PTCL-NOS, } \\
\text { peripheral T-cell lymphoma-not otherwise specified; AITL, angioimmunoblastic T-cell lymphoma; ALCL, } \\
\text { anaplastic large cell lymphoma; ALK, anaplastic lymphoma kinase; ECOG PS, Eastern Cooperative } \\
\text { Oncology Group performance status; LDH, lactate dehydrogenase; EN, extranodal involvement; BM, } \\
\text { bone marrow; IPI, International Prognostic Index; PIT, prognostic index for PTCL-unspecified; CHO(E)P, } \\
\text { cyclophosphamide, doxorubicin, vincristine, and prednisone (plus etoposide); ICED, ifosfamide, } \\
\text { carboplatin, etoposide, dexamethasone; IMEP, ifosfamide, methotrexate, etoposide, prednisone; } \\
\text { ESHAOx, etoposide, methylprednisolone, high-dose cytarabine, oxaliplatin; CR, complete response; PR, } \\
\text { partial response; SD, stable disease; PD, progressive disease; HSCT, hematopoietic stem cell } \\
\text { transplantation }\end{array}$} \\
\hline
\end{tabular}

\section{Response and survival outcomes according to the biomarkers}

The responses after induction chemotherapy are presented in Table 1, which shows that the high CAR group had a significantly lower rate of CR than the low CAR group ( $32.0 \% \mathrm{vs.} 76.5 \%, \mathrm{p}<0.001)$. With a median follow-up of 57.5 months, the high CAR group had significantly lower 5 -year PFS (6.6\% vs. $43.8 \%$, $p<0.0001)$ and OS $(20.2 \%$ vs. $62.2 \%, p<0.0001)$ rates (Fig. 1$)$. When we classified patients into low and high IPI groups (0-2 vs. $3-5)$, the high IPI group showed significantly poorer 5 -year PFS $(11.3 \%$ vs. $52.2 \%$, p $<0.0001)$ and OS $(21.1 \%$ vs. $73.4 \%, p<0.0001)$ rates (Fig. 2$)$. We also dichotomized patients with PIT (02 vs. $3-4)$ and T cell score ( $0-1$ vs. $2-4)$, and the results were statistically significant for both PFS and OS (Figs. 3 and 4). We applied the same method to designate the appropriate cutoff values for the other biomarkers, including beta-2 microglobulin (3.73), NLR (2.479), AGR (1.338), PLR (74.9), and ferritin (602.5). All results showed statistically significant differences in survival outcomes, except for AGR for PFS (Supplementary Figs. 1-5). During the follow-up period, 32 deaths occurred. Of these, 19 deaths were related to disease progression, and eight deaths were due to infectious conditions. One patient died of hemophagocytic lymphohistiocytosis, which occurred immediately after disease progression. Other causes of death included acute myocardial infarction, aspiration, cerebral infarction, and hepatic failure.

\section{Univariable and multivariable analyses of survival outcomes according to CAR}


The results of the univariate analysis for PFS and OS demonstrated that most components of the IPI and PIT were significantly associated with survival outcomes. High CAR was also significantly related to both PFS (HR: 5.12, 95\% Cl 2.74-9.59, p<0.001) and OS (HR: 3.85, 95\% Cl 2.13-6.97, p<0.001) (Table 2). After adjustment for components of the IPI, PIT, and T cell score, high CAR remained a significant factor for both PFS (HR: 2.83, 95\% Cl 1.34-5.95, p=0.006) and OS (HR 3.02, 95\% Cl 1.23-7.43, p=0.016) (Table 3).

Table 2

Univariable analyses of progression-free survival and overall survival in patients with peripheral T-cell lymphoma

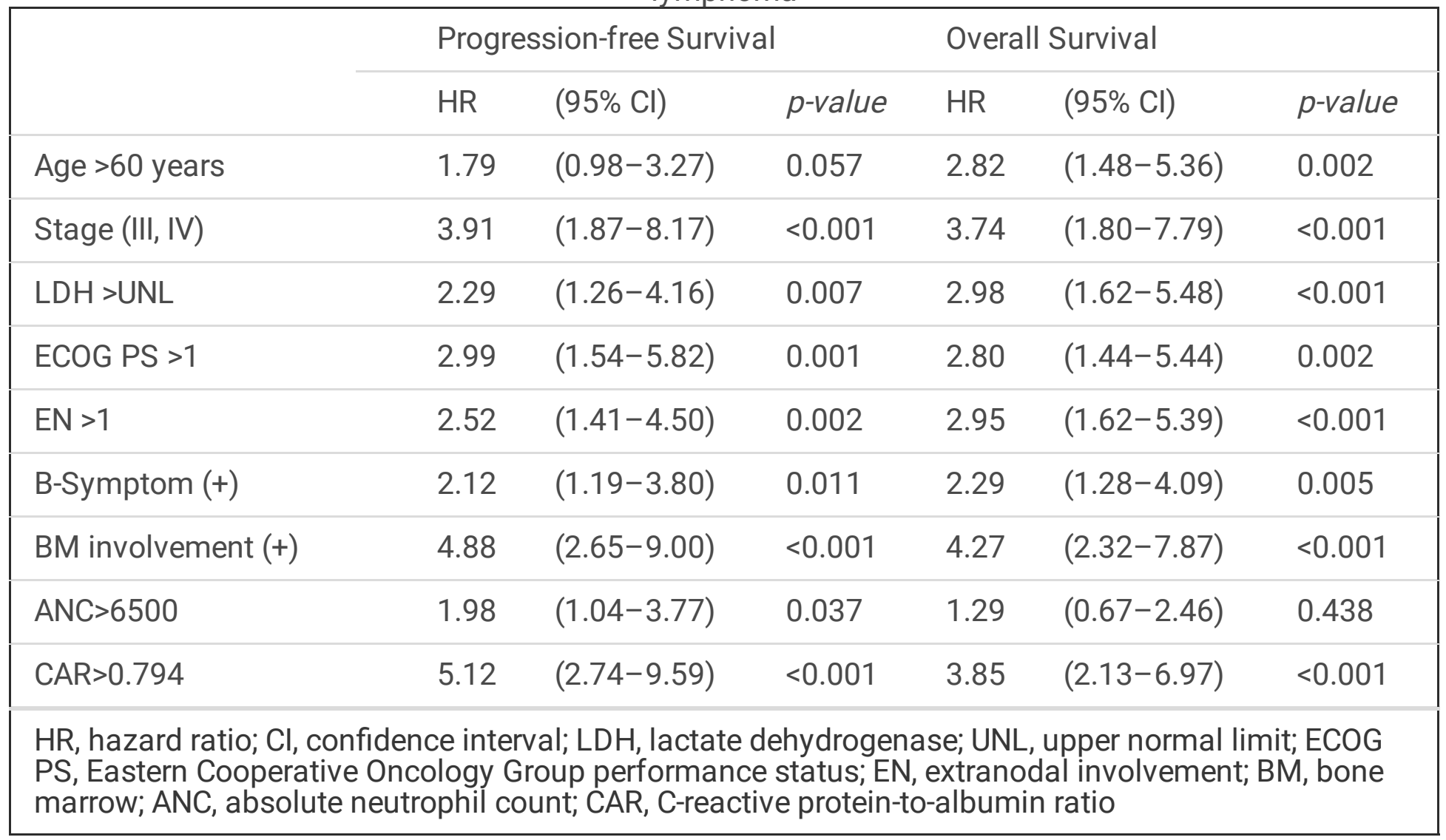


Table 3

Multivariable analyses of progression-free survival and overall survival in patients with peripheral T-cell lymphoma

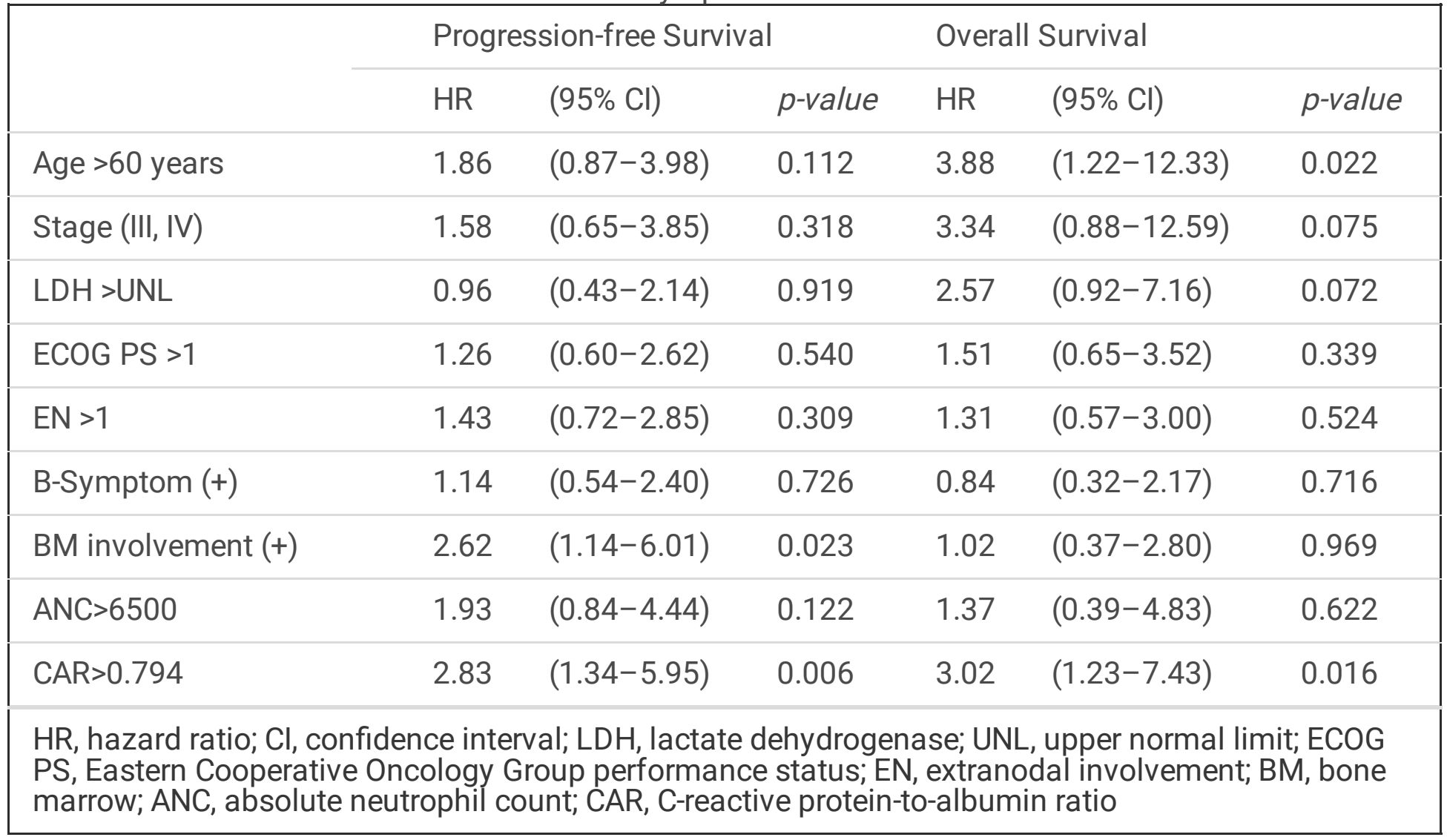

To compare the statistical significance of CAR directly to IPI, PIT, and T cell scores, we performed multivariable Cox analyses separately. After adjusting for the IPI, high CAR was significantly associated with inferior PFS (HR: 3.79, 95\% Cl 1.94-7.39, p<0.001) and OS (HR: 2.85, 95\% Cl 1.32-6.16, p=0.008), with a high IPI (HR: $3.23,95 \% \mathrm{Cl} 1.68-6.22$, p<0.001 for PFS; HR: 4.97, 95\% Cl 2.05-12.00, $p<0.001$ for OS) (Table 4). After adjusting for the PIT, both high CAR and PIT presented with significant PFS (HR: 4.20, 95\% Cl 2.14-8.23, $p<0.001$ for high CAR; HR: 3.42, 95\% Cl 1.79-6.56, $p<0.001$ for PIT $\geq 2$ ) and OS (HR: $3.14,95 \% \mathrm{Cl} 1.41-6.99, \mathrm{p}=0.005$ for high CAR; HR: 3.54, 95\% Cl 1.60-7.82, $\mathrm{p}=0.002$ for $\mathrm{PIT} \geq 2$ ). In regard to T cell score, high CAR remained a significant factor for both PFS (HR: 4.01, 95\% Cl 2.04-7.86, $p<0.001$ ) and OS (HR: 2.97, 95\% $\mathrm{Cl} 1.33-6.64, \mathrm{p}=0.008$ ). After adjusting for IPI, PIT, and T cell score, high CAR was significantly associated with PFS (HR: $3.69,95 \% \mathrm{Cl} 1.87-7.26, \mathrm{p}<0.001)$ and OS (HR: $2.33,95 \% \mathrm{Cl} 1.07-$ $5.09, p=0.034)$. 
Table 4

Multivariable analyses of progression-free survival and overall survival according to IPI, PIT, T cell score, and CAR

\begin{tabular}{|c|c|c|c|c|c|c|}
\hline & Progr & sion-free Surv & & Overa & Survival & \\
\hline & $\mathrm{HR}$ & $(95 \% \mathrm{Cl})$ & $p$-value & $\mathrm{HR}$ & $(95 \% \mathrm{Cl})$ & $p$-value \\
\hline $\mid \mathrm{PI} \geq 3$ & 3.23 & $(1.68-6.22)$ & $<0.001$ & 4.97 & $(2.05-12.00)$ & $<0.001$ \\
\hline CAR $>0.794$ & 3.79 & $(1.94-7.39)$ & $<0.001$ & 2.85 & $(1.32-6.16)$ & 0.008 \\
\hline $\mathrm{PIT} \geq 3$ & 3.42 & $(1.79-6.56)$ & $<0.001$ & 3.54 & $(1.60-7.82)$ & 0.002 \\
\hline CAR $>0.794$ & 4.20 & $(2.14-8.23)$ & $<0.001$ & 3.14 & $(1.41-6.99)$ & 0.005 \\
\hline T cell score $\geq 2$ & 1.85 & $(0.98-3.49)$ & 0.059 & 3.19 & $(1.43-7.11)$ & 0.005 \\
\hline CAR>0.794 & 4.01 & $(2.04-7.86)$ & $<0.001$ & 2.97 & $(1.33-6.64)$ & 0.008 \\
\hline$|P| \geq 3$ & 2.18 & $(0.98-4.87)$ & 0.056 & 3.27 & $(1.15-9.31)$ & 0.027 \\
\hline $\mathrm{PIT} \geq 3$ & 1.92 & $(0.86-4.29)$ & 0.111 & 1.39 & $(0.57-3.39)$ & 0.473 \\
\hline $\mathrm{T}$ cell score $\geq 2$ & 1.23 & $(0.64-2.36)$ & 0.530 & 1.90 & $(0.82-4.38)$ & 0.134 \\
\hline CAR $>0.794$ & 3.69 & $(1.87-7.26)$ & $<0.001$ & 2.33 & $(1.07-5.09)$ & 0.034 \\
\hline
\end{tabular}

\section{Discussion}

In this study, we performed a retrospective analysis of blood-based biomarkers as prognostic factors in patients with newly diagnosed PTCL. The results demonstrated that pretreatment CAR predicted the clinical behavior of PTCL in terms of response to induction chemotherapy and survival outcomes. In particular, we compared the statistical significance of CAR to previously established prognostic indexes, including the IPI, PIT, and T cell score, and high CAR was significantly associated with poor PFS and OS after adjusting for IPI, PIT, and T cell scores separately and together. Additionally, the prognostic significance of other biomarkers such as beta-2 microglobulin, NLR, AGR, PLR, and ferritin were assessed and revealed clinically meaningful outcomes, as previously reported for other lymphomas[16-19].

Importantly, CRP is a well-known marker of infectious diseases. It is primarily synthesized in hepatocytes and acts as an acute inflammatory protein that increases up to 1,000-fold at sites of inflammation[20]. Furthermore, a relationship between elevated CRP levels and the development of cancer has been established. In fact, a previous study suggested that the association between CRP level and cancer risk may be due to three possibilities. First, elevated CRP levels may cause cancer. Second, cancer increases CRP levels. Third, inflammation induces both increased CRP levels and the development of cancer[21]. In terms of the tumor microenvironment, inflammatory conditions have been shown to be associated with 
elevated CRP levels and cancer biology[22]. Although the causality between CRP and cancer has yet to be further elucidated, it is indisputable that CRP might be a useful marker in malignancies.

Serum albumin is known as a nutritional marker, but its physiological role also includes an antiinflammatory function[23]. Notably, hypoalbuminemia has been independently associated with inflammatory conditions represented by elevated CRP levels, believed to be driven from inflammatoryinduced capillary leakage[24]. Low serum albumin levels might also be caused by the secretion of inflammatory cytokines, such as interleukin- 6 and tumor necrosis factor-a, which are released by cancer[25,26]. On the basis of these mechanisms, albumin has been suggested as an independent prognostic marker of malignancies[27, 28]. In summary, high levels of pretreatment CAR may reflect inflammatory conditions and/or malnutritional status, which could be the result of cancer development or be due to a high degree of comorbidity and frailty, which are directly associated with survival outcomes in malignancies[29, 30].

Although high CAR was independent of previously known prognostic indexes, IPI and PIT were robust in our analyses. The T cell score was also significantly associated with OS in our patients. Taken together, our results showed that these previously established markers were effective in predicting clinical outcomes in PTCL and identifying CAR as another valuable marker considering its simplicity and objectivity. We also evaluated other biomarkers including beta-2 microglobulin, NLR, AGR, PLR, and ferritin. The analyses of these novel markers suggested that they could be used as simple and objective biomarkers of CAR. Although we did not include other possible prognostic markers, such as the EpsteinBarr virus and Ki-67 index due to the retrospective nature of our study, these markers have also been suggested to predict clinical outcomes[31, 32]. Additionally, gene expression profiles have been used in patients with diffuse large B-cell lymphoma as a prognostic marker[33]. However, while the usefulness of gene expression profiles in the prognostication of PTCL has been suggested, their use is still limited to defining prognosis[6]. Recently, brentuximab vedotin, an antibody-drug conjugate composed of an antiCD30 monoclonal antibody conjugated with a microtubule-disrupting drug, was approved as a first-line treatment for CD30-positive PTCL[34]. Importantly, other drugs such as pralatrexate, belinostat, or romidepsin, are available for the initial therapy of PTCL[1]. Therefore, the use of these indexes to predict clinical outcomes precisely in the era of novel agents warrants further attention.

Our study has several limitations. First, we included a substantial number of cases because of the relatively low incidence of PTCL. However, the number of cases was still limited to separate analyses for each type of PTCL. Second, the induction regimens were also heterogeneous due to the retrospective nature of the study and may be a possible confounding factor for survival outcomes, although this is unlikely given that no significant differences were detected in the selection of initial therapy. In addition, we used our own cutoff values of biomarkers, which we could not validate in the independent cohort due to the limited number of cases. Thus, appropriate cutoff values for each biomarker should be validated in future studies. However, it is obvious that the blood-based biomarkers that we investigated have the potential to be clinically meaningful prognostic indexes. Therefore, further investigations for cutoff values are warranted for these biomarkers to maximize clinical availability.

Page 13/20 
In conclusion, elevated CAR was associated with a worse response to treatment and poor survival outcome in patients with PTCL. CAR might play a complementary role in predicting prognosis in patients with PTCL, considering its simplicity, objectivity, and easy accessibility.

\section{Declarations}

\section{Acknowledgments}

This research was supported by the National Cancer Center research grant (no. 2110490-1).

\section{Author Contributions}

J.J. and J.Y.H. designed the study, collected the datasets, and drafted the manuscript. E.L., H.L., and M.H.C. participated in the data collection and drafted the manuscript. J.P. performed statistical analyses. H.E. analyzed the results and drafted the manuscript. All authors approved the final manuscript.

\section{Competing Interests:}

The authors declare no competing interests.

\section{Data Availability Statement:}

The datasets for this study are available upon request from the corresponding author.

\section{References}

1. Laribi, K., Alani, M., Truong, C. \& Baugier de Materre, A. Recent advances in the treatment of peripheral T-cell lymphoma. Oncologist 23, 1039-1053 (2018). 10.1634/theoncologist.2017-0524.

2. Numata, A. et al. Long-term outcomes of autologous PBSCT for peripheral T-cell lymphoma: retrospective analysis of the experience of the Fukuoka BMT group. Bone Marrow Transplant. 45, 311-316 (2010). 10.1038/bmt.2009.165.

3. Schmitz, N. \& de Leval, L. How I manage peripheral T-cell lymphoma, not otherwise specified and angioimmunoblastic T-cell lymphoma: current practice and a glimpse into the future. Br. J. Haematol. 176, 851-866 (2017). 10.1111/bjh.14473.

4. Savage, K. J. et al. ALK- anaplastic large-cell lymphoma is clinically and immunophenotypically different from both ALK+ ALCL and peripheral T-cell lymphoma, not otherwise specified: report from the International peripheral T-Cell Lymphoma Project. Blood 111, 5496-5504 (2008). 10.1182/blood2008-01-134270. 
5. Weisenburger, D. D. et al. Peripheral T-cell lymphoma, not otherwise specified: a report of 340 cases from the International peripheral T-cell Lymphoma Project. Blood 117, 3402-3408 (2011). 10.1182/blood-2010-09-310342.

6. Piccaluga, P. P. et al. Prognostic markers in peripheral T-cell lymphoma. Curr. Hematol. Malig. Rep. 5, 222-228 (2010). 10.1007/s11899-010-0062-x.

7. Gallamini, A., Stelitano, C. \& Calvi, R. Peripheral T-cell lymphoma unspecified (PTCL-U): a new prognostic model from a retrospective multicentric clinical study. Blood 103, 2474-2479 (2004). 10.1182/blood-2003-09-3080.

8. Federico, M. et al. Peripheral T cell lymphoma, not otherwise specified (PTCL-NOS). A new prognostic model developed by the International T cell Project Network. Br. J. Haematol. 181, 760-769 (2018). 10.1111/bjh.15258.

9. $\mathrm{Xu}, \mathrm{H}$. J. et al. The prognostic value of C-reactive protein/albumin ratio in human malignancies: an updated meta-analysis. Onco Targets Ther. 3059-3070 (2017). 10.2147/OTT.S137002.

10. Jung, J. et al. High level of pre-treatment C-reactive protein to albumin ratio predicts inferior prognosis in diffuse large B-cell lymphoma. Sci. Rep. 11, 2674 (2021). 10.1038/s41598-021-82087-6.

11. Di, Q. S. et al. High C-reactive protein to albumin ratio predicts inferior clinical outcomes in extranodal natural killer T-cell lymphoma. Dose-Response 18:1559325820917824 (2020). $10.1177 / 1559325820917824$.

12. Yang, J. et al. The prognostic value of blood-based biomarkers in patients With testicular diffuse large B-cell lymphoma. Front. Oncol. 9, 1392 (2019). 10.3389/fonc.2019.01392.

13. Mu, S. et al. Prognostic role of neutrophil-to-lymphocyte ratio in diffuse large B cell lymphoma patients: an updated dose-response meta-analysis. Cancer Cell Int. 18, 119 (2018). 10.1186/s12935018-0609-9.

14. Cheson, B. D. et al. Recommendations for initial evaluation, staging, and response assessment of Hodgkin and non-Hodgkin lymphoma: the Lugano classification. J. Clin. Oncol. Off. J. Am. Soc. Clin. Oncol. 32, 3059-3067 (2014). 10.1200/JC0.2013.54.8800.

15. Budczies, J. et al. Cutoff Finder: a comprehensive and straightforward Web application enabling rapid biomarker cutoff optimization. PLOS ONE 7, e51862 (2012). 10.1371/journal.pone.0051862.

16. Bento, L. et al. New prognosis score including absolute lymphocyte/monocyte ratio, red blood cell distribution width and beta-2 microglobulin in patients with diffuse large B-cell lymphoma treated with R-CHOP: Spanish Lymphoma Group Experience (GELTAMO). Br. J. Haematol. 188, 888-897 (2020). 10.1111/bjh.16263.

17. Jung, J. et al. Prognostic role of the neutrophil-to-lymphocyte ratio in patients with primary central nervous system lymphoma. Oncotarget 8, 74975-74986 (2017). 10.18632/oncotarget.20480.

18. $\mathrm{Bi}, \mathrm{X}$. W. et al. The pretreatment albumin to globulin ratio predicts survival in patients with natural killer/T-cell lymphoma. PeerJ 4, e1742 (2016). 10.7717/peerj.1742.

19. Zhao, Y. et al. The prognostic value of platelet-lymphocyte ratio and neutrophil-lymphocyte ratio in the treatment response and survival of patients with peripheral T-cell lymphoma. Leuk. Lymphoma 
61, 623-630 (2020). 10.1080/10428194.2019.1700244.

20. Sproston, N. R. \& Ashworth, J. J. Role of C-reactive protein at sites of inflammation and infection. Front. Immunol. 9, 754 (2018). 10.3389/fimmu.2018.00754.

21. Allin, K. H. \& Nordestgaard, B. G. Elevated C-reactive protein in the diagnosis, prognosis, and cause of cancer. Crit. Rev. Clin. Lab. Sci. 48, 155-170 (2011). 10.3109/10408363.2011.599831.

22. Nakayama, T. et al. Higher serum C-reactive protein level represents the immunosuppressive tumor microenvironment in patients With clear cell renal cell carcinoma. Clin. Genitourin. Cancer 16, e1151-e1158 (2018). 10.1016/j.clgc.2018.07.027.

23. Arques, S. Human serum albumin in cardiovascular diseases. Eur. J. Intern. Med. 52, 8-12 (2018). 10.1016/j.ejim.2018.04.014.

24. Eckart, A. et al. Relationship of nutritional status, inflammation, and serum albumin levels During acute illness: A prospective study. Am. J. Med. 133, 713-722.e7 (2020). 10.1016/j.amjmed.2019.10.031.

25. Balkwill, F. \& Mantovani, A. Inflammation and cancer: back to Virchow? Lancet 357, 539-545 (2001). 10.1016/S0140-6736(00)04046-0.

26. Brenner, D. A., Buck, M., Feitelberg, S. P. \& Chojkier, M. Tumor necrosis factor-alpha inhibits albumin gene expression in a murine model of cachexia. J. Clin. Invest. 85, 248-255 (1990). 10.1172/JCl114419.

27. Wei, Y. et al. Albumin improves stratification in the low IPI risk patients with diffuse large B-cell lymphoma. Int. J. Hematol. 111, 681-685 (2020). 10.1007/s12185-020-02818-9.

28. Gupta, D. \& Lis, C. G. Pretreatment serum albumin as a predictor of cancer survival: a systematic review of the epidemiological literature. Nutr. J. 9, 69 (2010). 10.1186/1475-2891-9-69.

29. Boakye, D. et al. Impact of comorbidity and frailty on prognosis in colorectal cancer patients: A systematic review and meta-analysis. Cancer Treat. Rev. 64, 30-39 (2018). 10.1016/j.ctrv.2018.02.003.

30. Morrison, V. A. et al. Diffuse large B-cell lymphoma in the elderly: impact of prognosis, comorbidities, geriatric assessment, and supportive care on clinical practice. An International Society of Geriatric Oncology (SIOG) expert position paper. J. Geriatr. Oncol. 6, 141-152 (2015). 10.1016/j.jgo.2014.11.004.

31. Went, P. et al. Marker expression in peripheral T-cell lymphoma: a proposed clinical-pathologic prognostic score. J. Clin. Oncol. Off. J. Am. Soc. Clin. Oncol. 24, 2472-2479 (2006). 10.1200/JC0.2005.03.6327.

32. Dupuis, J. et al. Prognostic significance of Epstein-Barr virus in nodal peripheral T-cell lymphoma, unspecified: A Groupe d'Etude des Lymphomes de l'Adulte (GELA) study. Blood 108, 4163-4169. 10.1182/blood-2006-04-017632.

33. Rosenwald, A. \& Staudt, L. M. Gene expression profiling of diffuse large B-cell lymphoma. Leuk. Lymphoma 44 Suppl 3, S41-S47 (2003). 10.1080/10428190310001623775. 
34. Horwitz, S. et al. Brentuximab vedotin with chemotherapy for CD30-positive peripheral T-cell lymphoma (ECHELON-2): a global, double-blind, randomised, phase 3 trial. Lancet 393, 229-240 (2019). 10.1016/S0140-6736(18)32984-2.

\section{Figures}
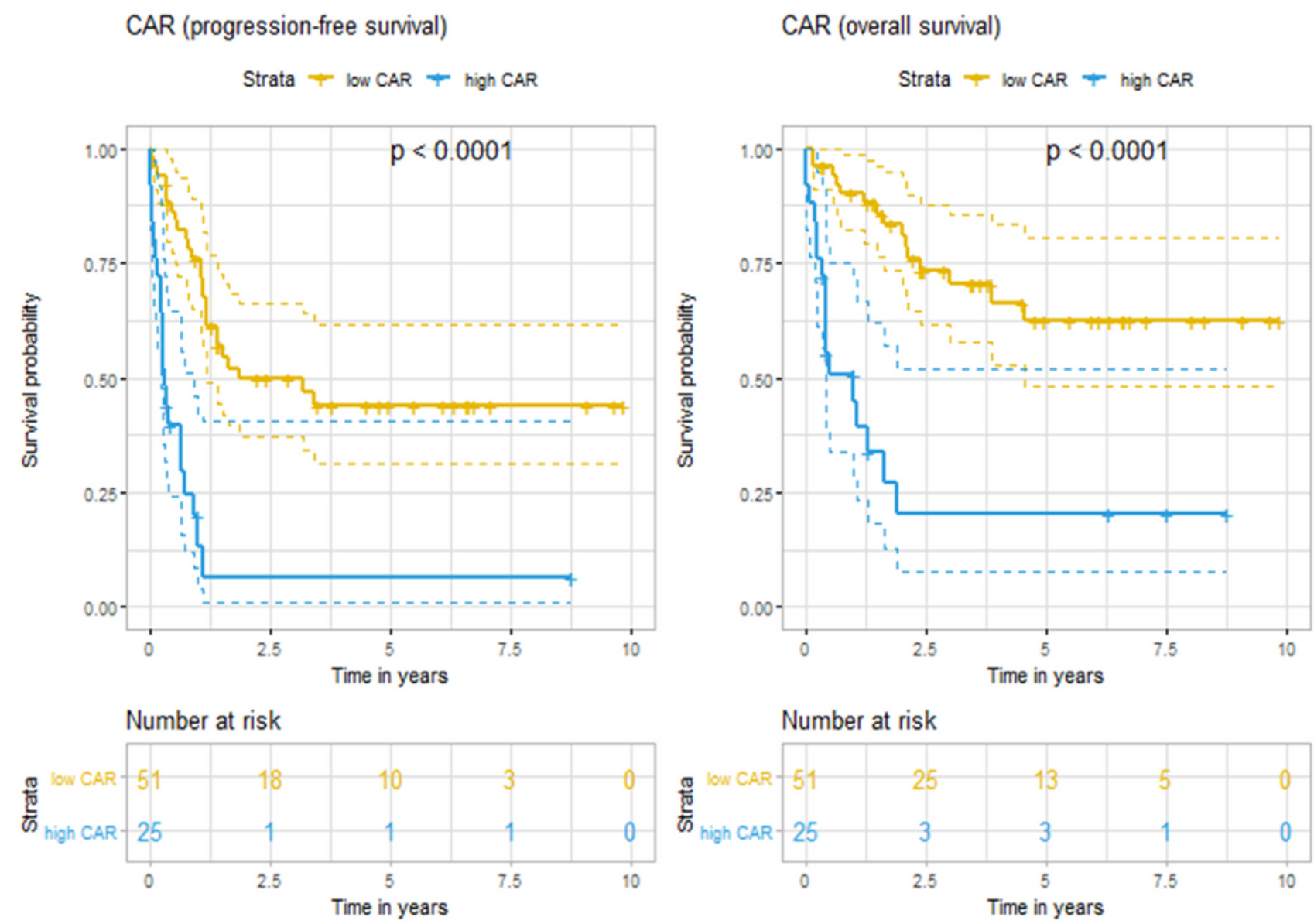

\section{Figure 1}

Kaplan-Meier curves for progression-free survival and overall survival according to the C-reactive proteinto-albumin ratio (CAR) at the diagnosis of peripheral T-cell lymphoma. 
IPI (progression-free survival)

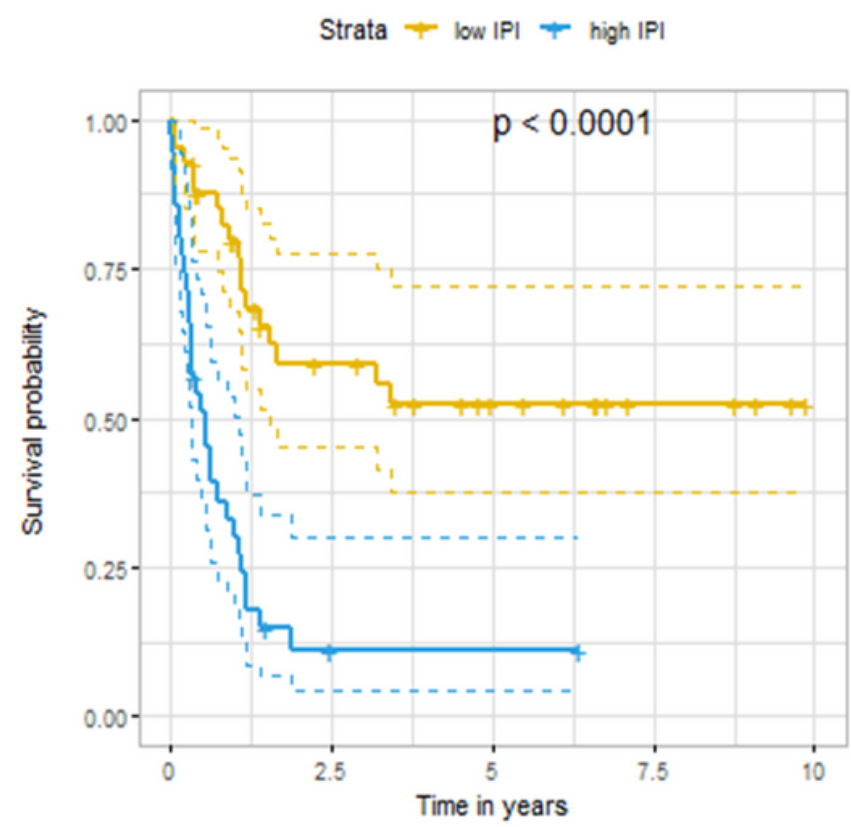

Number at risk

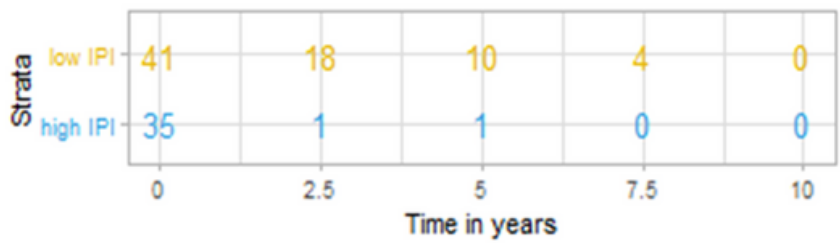

IPI (overall survival)

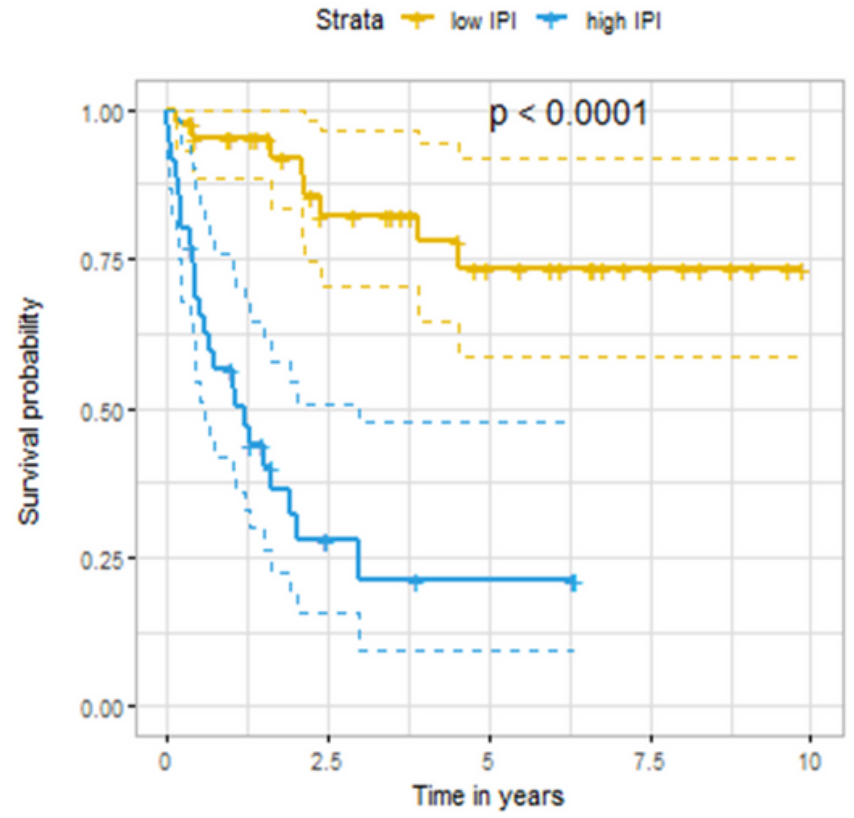

Number at risk

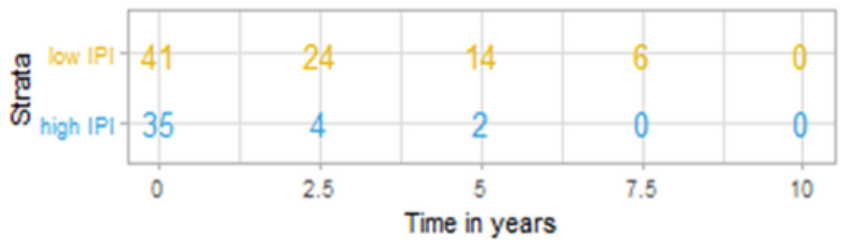

Figure 2

Kaplan-Meier curves for progression-free survival and overall survival according to the International Prognostic Index (IPI) at the diagnosis of peripheral T-cell lymphoma. 
PIT (progression-free survival)

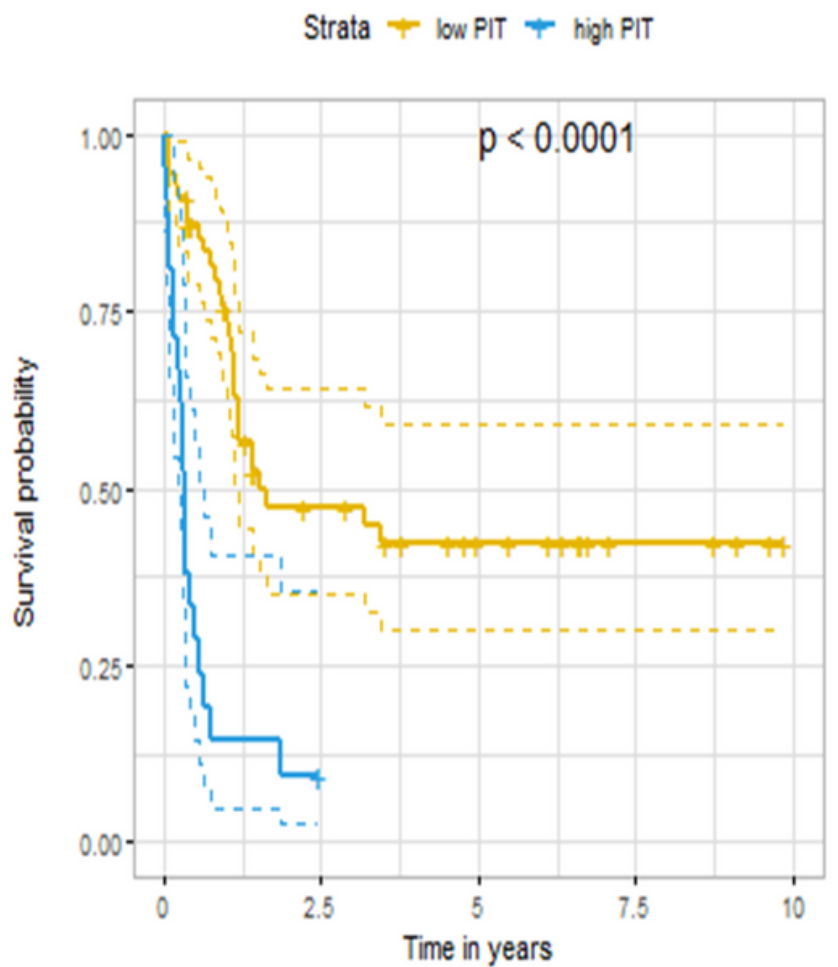

Number at risk

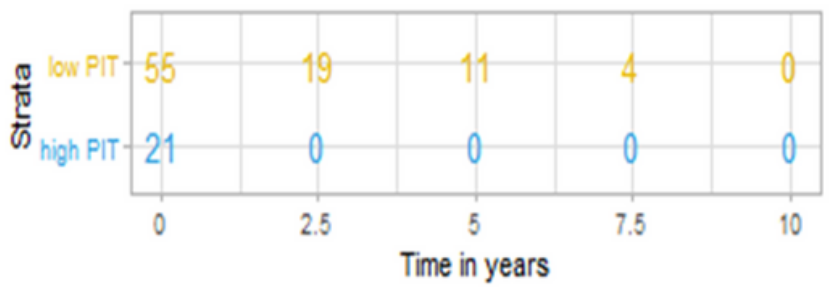

PIT (overall sunival)

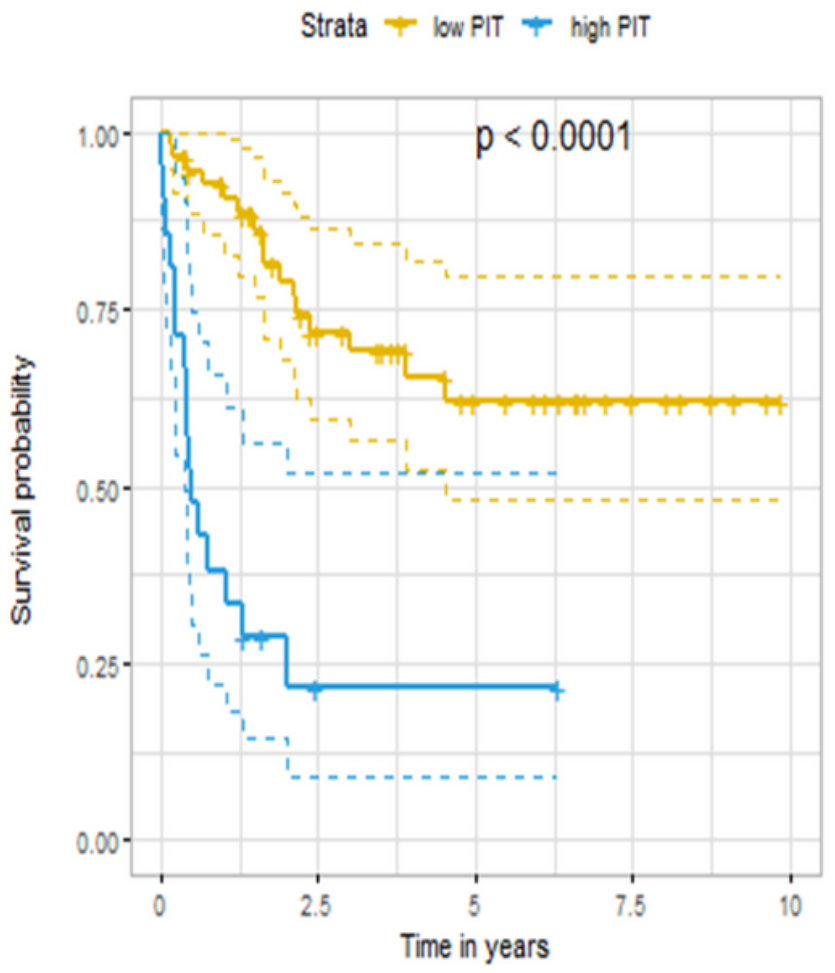

Number at risk

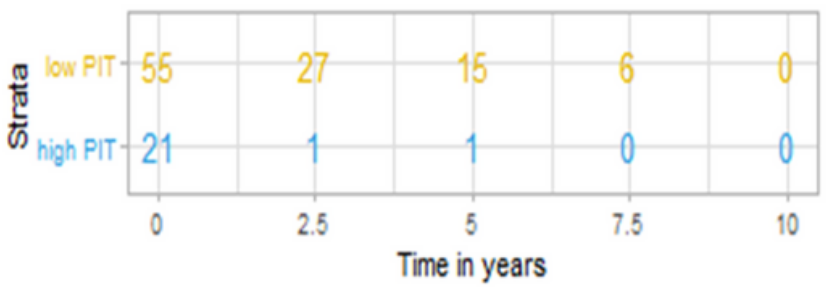

\section{Figure 3}

Kaplan-Meier curves for progression-free survival and overall survival according to the Prognostic Index for PTCL-unspecified at the diagnosis of peripheral T-cell lymphoma. 

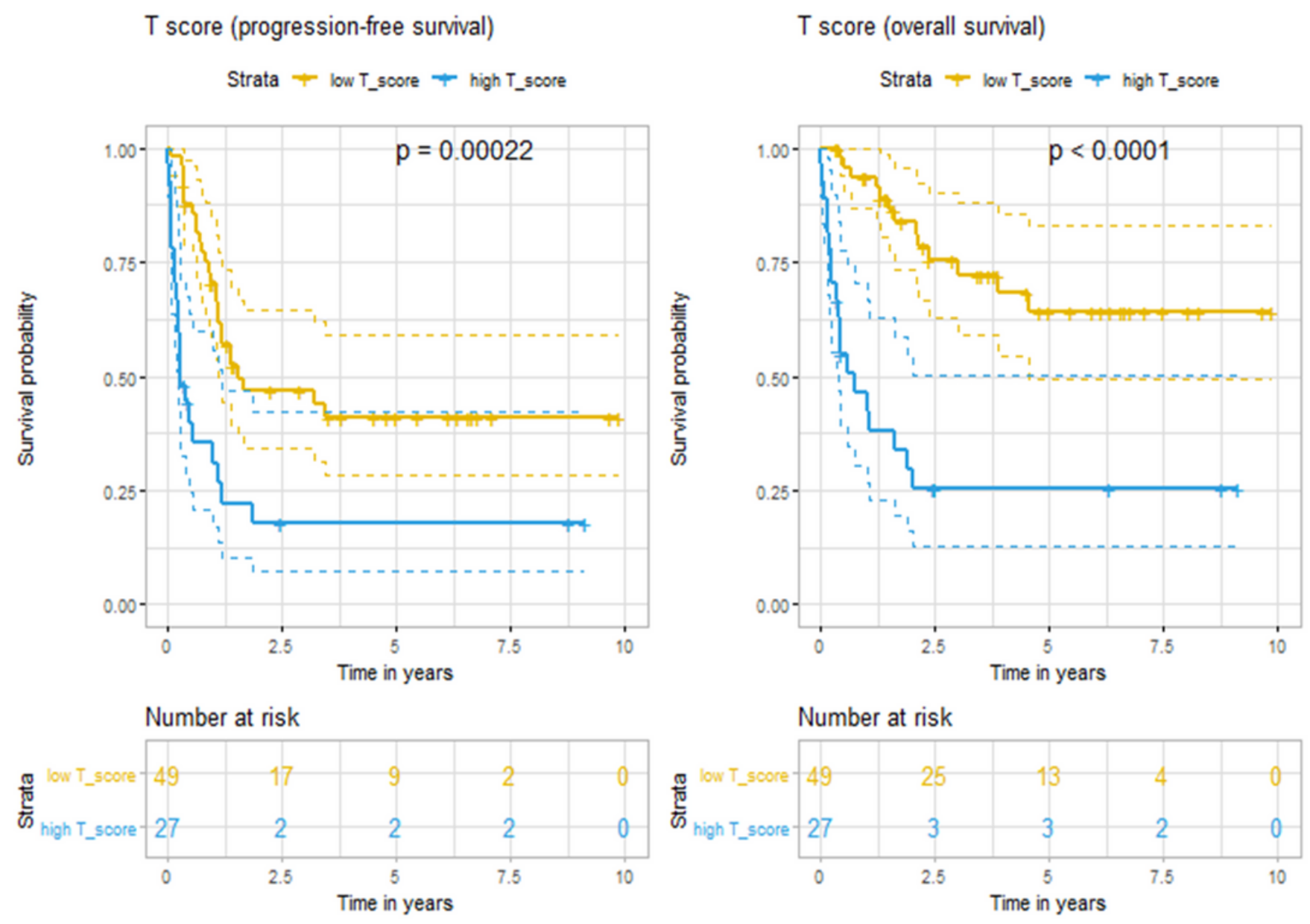

\section{Figure 4}

Kaplan-Meier curves for progression-free survival and overall survival according to the $T$ cell score developed by the International T cell Project Network at the diagnosis of peripheral T-cell lymphoma.

\section{Supplementary Files}

This is a list of supplementary files associated with this preprint. Click to download.

- CARPTCLsupplementarySRver1.0.docx 